\title{
Surgical Treatment for Atlanto-Occipital Subluxation due to Destructive Spondyloarthropathy in a Patient Undergoing Long-Term Hemodialysis
}

\author{
Keiji Wada, Yasuaki Murata, Yoshiharu Kato \\ Department of Orthopedics, Tokyo Women's Medical University, Tokyo, Japan
}

\begin{abstract}
Destructive spondyloarthropathy (DSA) has been reported in patients undergoing long-term hemodialysis. Cervical spinal lesions, including those of the upper cervical spine, are reported to be some of the most common. To our knowledge, we report for the first time, a case of atlanto-occipital subluxation requiring surgical treatment due to severe myelopathy and nuchal pain in a patient undergoing long-term hemodialysis. The patient was a 66-year-old woman who had undergone hemodialysis for 40 years. She visited our hospital due to an acute progression of gait disturbance and severe nuchal pain. Computed tomography showed posterior subluxation of the atlanto-occipital joints. DSA was also observed in the lower cervical spine. Magnetic resonance imaging showed spinal canal stenosis at both the upper and lower cervical levels. We performed Oc-C7 fixation, C1 laminectomy, and C3-C7 laminoplasty. We first recognized that the atlanto-occipital subluxation was caused by the extremely long-term, in this case, 40 years, hemodialysis.
\end{abstract}

Keywords: Hemodialysis; Destructive spondyloarthropathy; Atlanto-occipital subluxation

\section{Introduction}

Destructive spondyloarthropathy (DSA) has been reported in patients undergoing long-term hemodialysis, and one of the sites most commonly affected is the cervical spine, including the upper cervical spine [1,2]. Besides DSA, amyloid deposition in the spinal canal and dural calcification sometimes compresses the spinal cord, causing radiculopathy or myelopathy [3]. Pseudotumors around the dens and atlanto-axial DSA are among the lesions that can occur in the upper cervical spine, causing severe myelopathy or nuchal pain and often requiring surgical treatment $[4,5]$. Gerster et al. [6] reported on
DSA changes such as bone cysts and joint space narrowing in the atlanto-occipital joints of patients undergoing long-term hemodialysis. To our knowledge, this is the first report describing atlanto-occipital subluxation requiring surgical treatment in a patient undergoing long-term hemodialysis.

\section{Case Report}

A 66-year-old woman presented to our hospital with severe bilateral hand clumsiness, gait disturbance, and nuchal pain. She had undergone hemodialysis for 40 years due to glomerulonephritis. A fusion surgery had been

Received Jul 8, 2014; Revised Aug 12, 2014; Accepted Sep 9, 2014

Corresponding author: Keiji Wada

Department of Orthopedics, Tokyo Women's Medical University,

8-1 Kawada-cho, Shinjuku-ku, Tokyo 162-8666, Japan

Tel: +81-3-3353-8111, Fax: +81-3-3354-7360, E-mail: keijiwadajp@yahoo.co.jp 
performed in her lumbar spine due to DSA 8 years earlier. The patient's bilateral hand clumsiness and gait had gradually deteriorated in the month prior to her presentation at our hospital. She had also experienced severe nuchal pain for the past 3 months. A neurological exam revealed that deep tendon reflexes in both upper extremities were markedly increased. The patellar tendon reflexes were also markedly increased bilaterally. Both Achilles tendon reflexes were decreased, probably due to the previous lumbar spine lesion. Manual muscle testing revealed generalized weakness (grade 4/5) throughout the extremities. Sensation was impaired below the upper cervical area. She had severe hand clumsiness and was barely able to walk due to severe spasticity of the lower extremities. She also reported severe nuchal pain. Based on the neurological findings, we concluded she had myelopathy below the upper cervical level. Her Japanese Orthopedics Association (JOA) score for cervical myelopathy was 1 out of a maximum score of 14 (3 points from the JOA total score of 17 is deducted for patients undergoing hemodialysis because of the difficulty in evaluating their bladder function) [7].

Sagittal computed tomography (CT) at the atlantooccipital joints levels showed bone cysts and posterior subluxation of the bilateral joints (Fig. 1A, B). Sagittal CT at the midline level showed the clivus located posterior to the odontoid process, suggesting posterior subluxation of the atlanto-occipital joints. DSA changes were also observed in the lower cervical spine (Fig. 1C). Magnetic resonance imaging (MRI) showed spinal canal stenosis at both the upper and lower cervical levels (Fig. 2).
We performed posterior Oc-C7 fixation, C1 posterior arch resection, and C3-C7 laminoplasty. A block of iliac bone graft was placed between $\mathrm{Oc}$ and $\mathrm{C} 2$. Bone graft was also placed between $\mathrm{C} 3$ and $\mathrm{C} 7$. Postoperative radi-

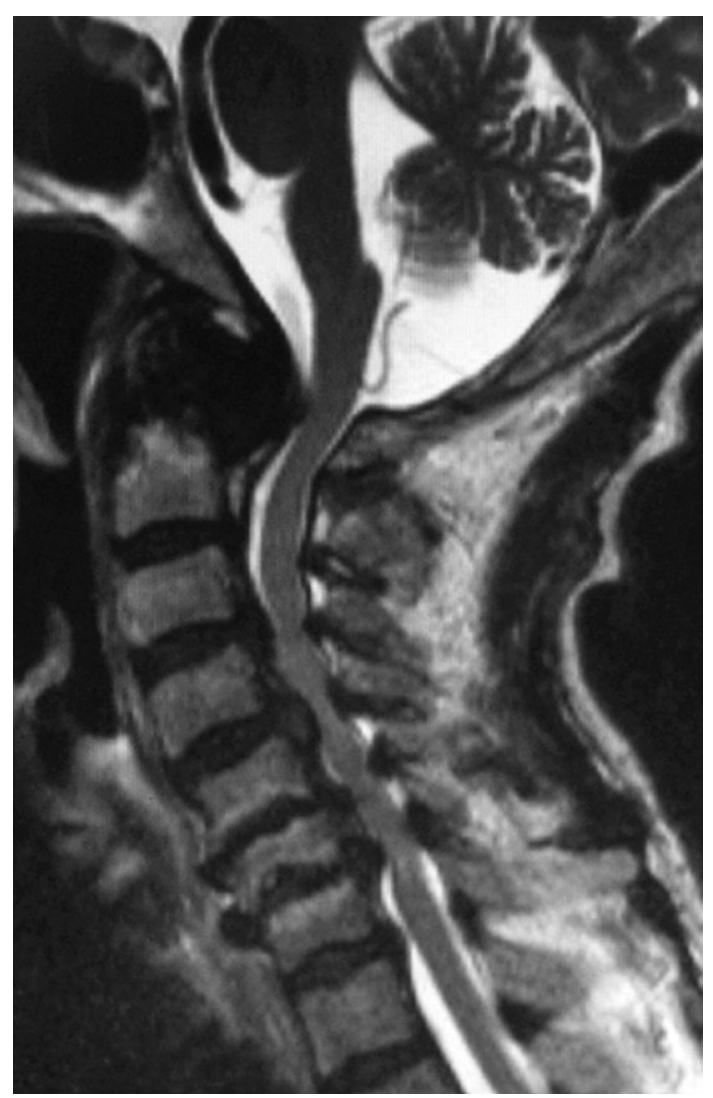

Fig. 2. Magnetic resonance imaging shows spinal canal stenosis at the upper cervical level and C3-C7.
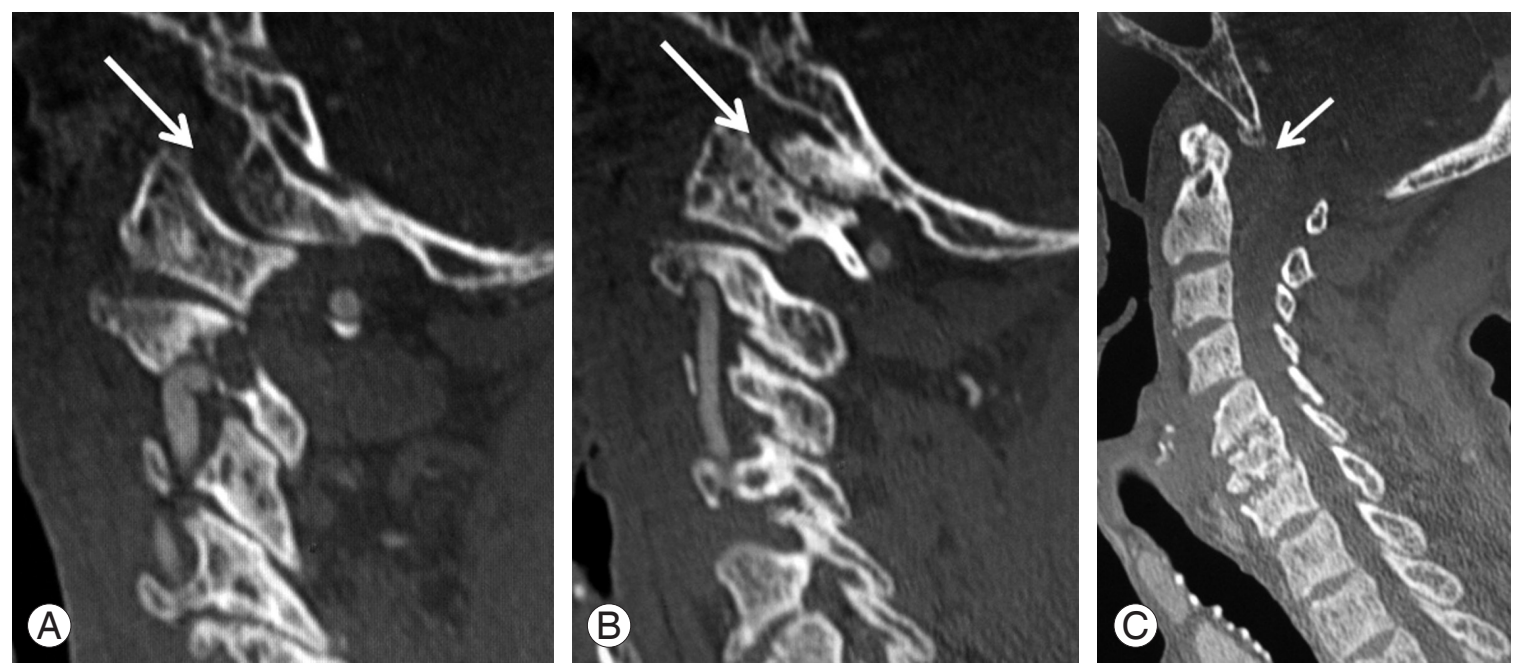

Fig. 1. Sagittal computer tomography shows the destruction and subluxation of the right (A) and left (B) atlanto-occipital joints (arrow) and the clivus (C) located posterior to the odontoid process (arrow) 
ography showed a good alignment of the cervical spine (Fig. 3A). A solid bone union, an expanded spinal canal, and reduction of the clivus (Fig. 3B) were observed on a postoperative CT 14 months after the surgery. Eighteen months after surgery, the patient's postoperative JOA score was 7 points and her nuchal pain had disappeared.

The patient was fully informed that her data would be submitted for publication, and she gave her consent.

\section{Discussion}

We encountered what may be the first reported case of atlanto-occipital subluxation in a patient undergoing long-term hemodialysis that could be treated surgically. The literature contains several reports describing upper cervical lesions in patients undergoing long-term hemodialysis, some of which were treated surgically for periodontoid pseudotumor [8,9]. Takeshima et al. [8] and Vignes et al. [9] both reported that C1 laminectomy and tumor resection are possible for pseudotumors located on the posterolateral side of the odontoid process.

Moreover, several reports concerning atlantoaxial DSA have been published [5,6]. Gerster et al. [6] reported that $38 \%$ of patients undergoing long-term hemodialysis showed upper cervical lesions, including cystic radiolucency in the $\mathrm{C} 1$ lateral mass, dens, and body of $\mathrm{C} 2$ on radiography. Since patients with myelopathy due to atlantoaxial DSA sometimes suffer fatal spinal cord injuries with minor trauma, surgery is required. Among the surgical treatments for atlantoaxial DSA described, Mikawa et al. [10] reported on the Brooks procedure with halo-vest immobilization and Kato et al. [5] performed a combined Magerl and Brooks procedure for an atlantoaxial DSA with satisfactory results. Odontoid fractures due to DSA can causes especially severe instability and have a higher risk of spinal cord injury, making fixation surgery mandatory.

Gerster et al. [6] reported that radiography showed atlanto-occipital joint changes in two of 23 patients undergoing long-term hemodialysis. Slight instability at the atlanto-occipital joints has also been documented in two of five patients with atlantoaxial DSA [10]. These reports indicate that long-term hemodialysis may cause atlantooccipital subluxation, resulting in severe myelopathy and nuchal pain, as in the present case. To our knowledge, this is the first report of atlanto-occipital subluxation requiring surgical treatment in a long-term hemodialysis patient.
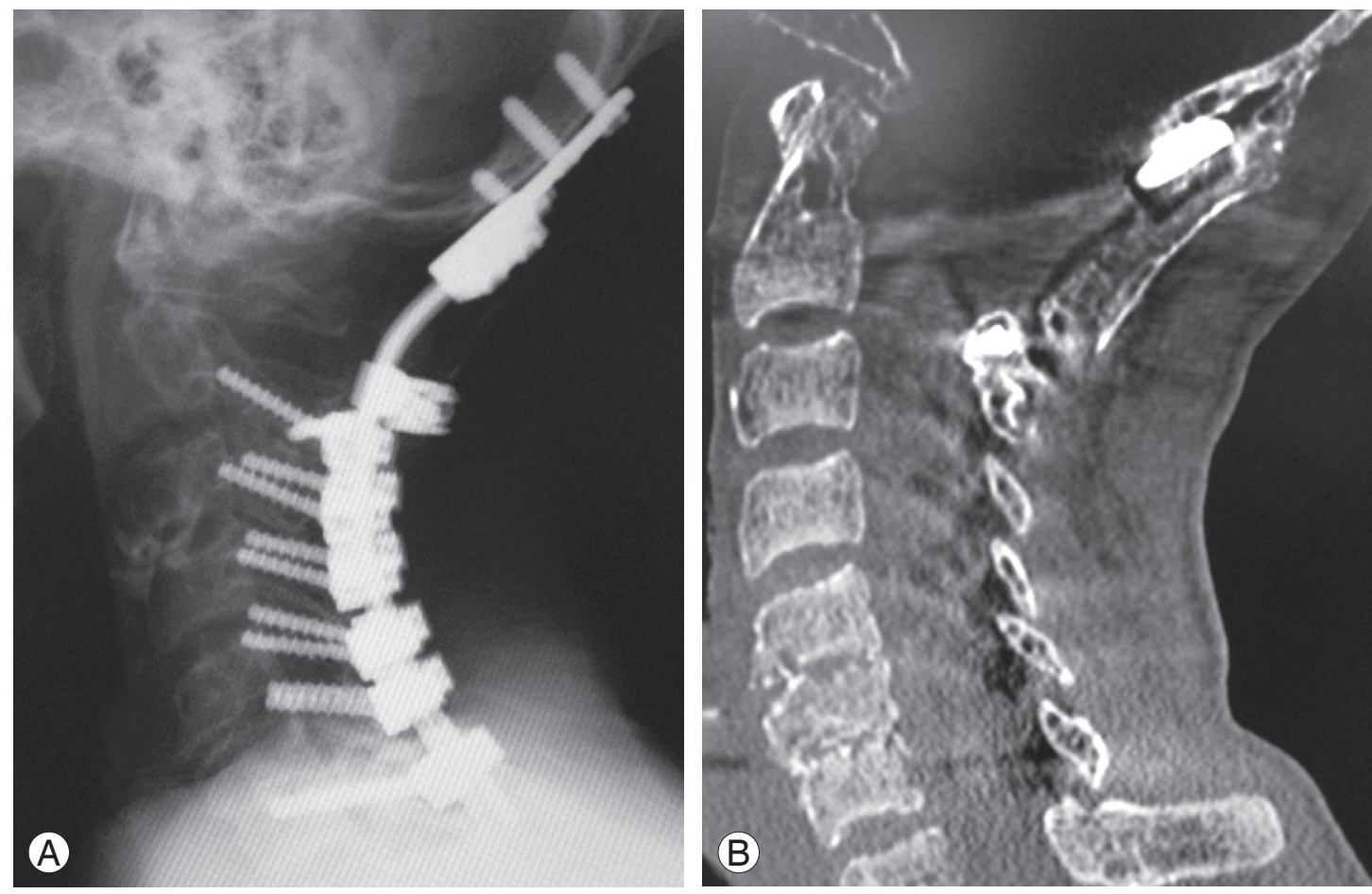

Fig. 3. (A) Lateral radiography at 14 months postoperatively. (B) Sagittal computer tomography at 12 months shows a solid bony union between the occiput and bone graft. 
Fortunately, our patient's myelopathy recovered completely following the occipitocervical fusion surgery, and no life-threatening complications occurred.

\section{Conflict of Interest}

No potential conflict of interest relevant to this article was reported.

\section{References}

1. Kuntz D, Naveau B, Bardin T, Drueke T, Treves R, Dryll A. Destructive spondylarthropathy in hemodialyzed patients: a new syndrome. Arthritis Rheum 1984;27:369-75.

2. Spinos P, Matzaroglou C, Partheni M, Deli A, Karanikolas M, Konstantinou D. Surgical management of cervical spondyloarthropathy in hemodialysis patients. Open Orthop J 2010;4:39-43.

3. Shiraishi T, Ikegami T, Okubo Y, Yato Y, Honda M. Cervical peridural calcification in patients undergoing long-term hemodialysis: report of two cases. J Neurosurg 2004;100:284-6.

4. Amoroso E, Vitale C, Silvestro A. Spinal-cord compression due to extradural amyloidosis of the cervico-occipital hinge, in a hemodialysed patient: a case report. J Neurosurg Sci 2001;45:120-4.

5. Kato Y, Kanaya K, Itoh T. Destructive spondyloarthropathy of the atlantoaxial joint with severe spinal cord compression in long-term hemodialysis patients. J Orthop Sci 2006;11:644-50.

6. Gerster JC, Carruzzo PA, Ginalski JM, Wauters JP. Cervicooccipital hinge changes during longterm hemodialysis. J Rheumatol 1989;16:1469-73.

7. Ogawa Y, Chiba K, Matsumoto M, et al. Long-term results after expansive open-door laminoplasty for the segmental-type of ossification of the posterior longitudinal ligament of the cervical spine: a comparison with nonsegmental-type lesions. J Neurosurg Spine 2005;3:198-204.

8. Takeshima Y, Kotsugi M, Park YS, Nakase H. Hemodialysis-related upper cervical extradural amyloidoma presenting with intractable radiculopathy. Eur Spine J 2012;21 Suppl 4:S463-6.

9. Vignes JR, Eimer S, Dupuy R, Donois E, Liguoro D. Beta(2)-Microglobulin amyloidosis caused spinal cord compression in a long-term haemodialysis patient. Spinal Cord 2007;45:322-6.

10. Mikawa Y, Yamaoka T, Watanabe R. Compression of the spinal cord due to destructive spondyloarthropathy of the atlanto-axial joints. J Bone Joint Surg Am 1996;78:1911-4. 